\title{
Alopecia universalis during treatment with leflunomide and adalimumab - Case report ${ }^{*}$
}

\author{
Rosana Lazzarini ${ }^{1}$ \\ Gabriela Cunha Capareli ${ }^{1}$ \\ Roberta Buense ${ }^{1}$ \\ Rute Facchini Lellis ${ }^{1}$
}

Abstract: Alopecia areata is a non-scarring form of alopecia that can be localized or widespread. Its etiology is unknown, but immunological factors are implicated in its pathogenesis. With the more frequent use of anti TNF$\alpha$ biologic drugs, some alopecia areata cases during their use have been described. We report a case of universal alopecia in a patient with rheumatoid arthritis while using adalimumab and leflunomide.

Keywords: Alopecia; Alopecia areata; Biological therapy; Rheumatoid arthritis

\section{INTRODUCTION}

Alopecia areata (AA) is a non-scarring form of alopecia, which affects approximately $1.7 \%$ of the population. ${ }^{1}$ The loss of hair may be localized or widespread and even affect the nail in some cases. Its etiology is unknown, but immunological factors have been implicated in its pathogenesis, due to the presence of inflammatory infiltrate of T-lymphocytes around hair follicles seen through histology, as well as the involvement of cytokines, including tumor necrosis factor alpha (TNF- $\alpha$ ).

With the more frequent usage of anti TNF- $\alpha$ biological drugs for the treatment of some diseases like rheumatoid arthritis, psoriasis, Chron's disease and ankylosing spondylitis, some AA cases after their use have been reported, suggesting a causal relationship between them.

We report a case of severe AA, known as alopecia universalis (AU), in a rheumatoid arthritis patient using Adalimumab and Leflunomide.

\section{CASE REPORT}

Female patient, 66 years old, with a history of rheumatoid arthritis and hypothyroidism, was referred to our clinic by the rheumatologist, with complaint of loss of all body hair and alterations in her fingernails for the past six months. She denied having similar symptoms before. She had been using
Adalimumab and Leflunomide for one year due to rheumatoid arthritis (RA), but as a result of alterations in hair and nails, her rheumatologist modified the therapeutic scheme, replacing adalimumab by etanercept and suspending leflunomide, with no clinical picture alteration, and then referred her to our clinic. During the dermatological exam she presented nonscarring alopecia of universal distribution, with presence of few hairs, thin and pigmented, around $3 \mathrm{~cm}$ long, on the scalp (Figure 1). All hand nails presented dystrophy with presence of cupuliform depressions (pitting) (Figure 2).

Dermoscopy of the scalp demonstrated presence of dystrophic hair and black dots, with some yellowish spots (Figure 3). A diagnostic hypothesis of AU triggered by usage of anti-TNF drug (adalimumab) and/or leflunomide was made and a biopsy of scalp was performed. The latest revealed presence of follicles with superficial, miniaturized hairs involved by a peribulbar perifollicular lymphocytic inflammatory infiltrate (Figure 4).

Topical treatment with minoxidil 5\% was initiated and the possibility of suspending etanercept was discussed with the rheumatologists. After 18 months without using the medications the patient progressed to an intense regrowth of scalp hair, but still with some alopecia areas (Figure 5).

\footnotetext{
Received on 08.07.2013.

Approved by the Advisory Board and accepted for publication on 05.08.2013

* Work performed at the Dermatology Clinic of Santa Casa de São Paulo - São Paulo (SP), Brazil.

Financial Support: none

Conflict of Interests: none

Santa Casa de São Paulo - São Paulo (SP), Brazil.
}

(C2014 by Anais Brasileiros de Dermatologia 

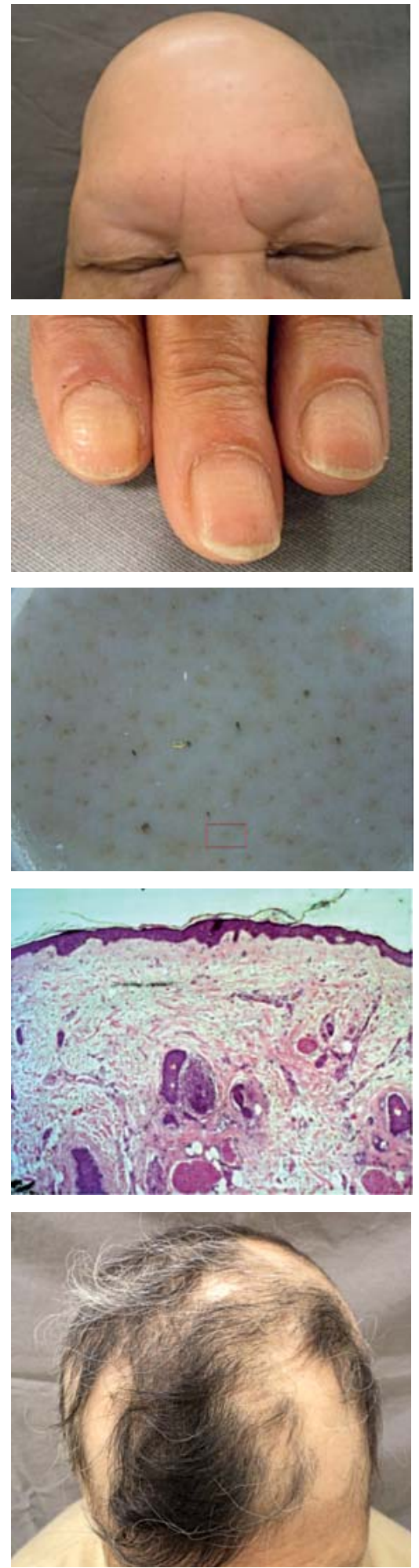

Figure 1:

Total absence of hairs on scalp, eyebrows and eyelashes

FiguRe 2:

Cupuliform depressions on fingernails and some linear hemorrhages

Figure 3:

Dermoscopy of scalp: black dots (yellow arrow) characterizing dystrophic hair and yellow dots (red rectangle), demonstrating activity of the disease

Figure 4:

Histopathology. Vertical section showing superficial, miniaturized hair follicles involved by a peribulbar and perifollicular lymphocytic inflammatory infiltrate on the dermis

FigURE 5: Hair regrowth after 18 months of follow-up and without use of drugs

\section{DISCUSSION}

The pathogenesis of AA remains uncertain, but it is believed that a complex autoimmune mechanism involving $\mathrm{T}$ lymphocytes and proinflammatory cytokines, like tumor necrosis factor alpha (TNF- $\alpha$ ), would be responsible for hair growth inhibition due to its inflammatory action on the hair bulb. Among the immunobiologicals, adalimumab and etanercept have as their action mechanism the selective inhibition of TNF- $\alpha$ and would be drugs with possible applicability for the treatment of AA. However, reports in the literature showed effects, not yet clarified, of these drugs on the hair bulb, promoting, paradoxically, hair loss.

Some reports in the literature show the onset of AA during or after usage of anti-TNF drugs and only one report described the disease being triggered in a patient using leflunomide alone. ${ }^{2}$ These reports show similar incidence between the sexes with development average of AA varying between 24-48h up to 208 weeks after starting medication use, making it difficult to establish a causal nexus.

Personal history of AA seems to be a facilitating factor for the onset of new lesions as reported by Bartels $^{3}$ and Posten. ${ }^{4}$ Suspension of medication intake seems to be fundamental for hair regrowth. ${ }^{5}$

Leflunomide is a drug used in the treatment of RA, which hinders the interaction of $\mathrm{T}$ cells with antigen-presenting ones. Its most common collateral effects encompass diarrhea, nausea, maculopapular exanthema, weight loss, increase in hepatic enzymes and transitory alopecia. The report that associates the use of this drug with AA informs that its suspension resulted in complete hair regrowth in the affected area ${ }^{6}$

Other drugs have been related to the onset of AA, among them, association of pegylated interferon, ribavirin and haloperidol, whose mechanisms are also not clear. ${ }^{7}$

In the presented case initial suspension of one of the drug (leflunomide) did not alter the process, making one suppose that the triggering factor still persisted. Replacing one TNF inhibitor by another did not contribute to improve the clinical picture, although no therapy had been instituted for AA treatment, at the time. However, around three months after suspending the use of the medication, the start of hair regrowth was observed, especially on the scalp. Notwithstanding it being a dermatosis of difficult treatment, some authors, like Tosti et cols showed that the suspension of these drugs, followed by specific therapy for AA, seems to be fundamental for hair regrowth. ${ }^{5}$ On the other hand, a recent publication by Zschoce e cols. reported a similar case, associated with the use of adalimumab, which after the introduction of etanercept did not cause the onset of new areas 
of alopecia. This fact made the authors suggest that the effect caused by a anti-TNF drug might not transfer to another of the same group, fact that was not observed in the case here described. ${ }^{8}$

Based on literature data and on the correlation between the onset of the clinical picture and the medication used we believe there is a clear relationship between both events. The patient is currently being followed-up at the rheumatology, where the possibility of no longer treating RA with anti-TNF medication is being evaluated, with methotrexate as an option. This substitution would favor AA therapy, for it is also an option for the treatment of this dermatosis. ${ }^{9}$

Due to the increasing number of patients under treatment with drugs that have anti-TNF properties, the knowledge and reporting of proportionally growing adverse effects turns out to be important. Furthermore, the recurrence or development of AA in patients under anti-TNF- $\alpha$ drugs makes it necessary to review the real importance of this cytokine and of $\mathrm{T}$ lymphocytes as important factors in the pathogenesis of AA, as well as the indication of biologicals for its therapy.

\section{REFERENCES}

1. Safavi KH, Muller SA, Suman VJ, Moshell AN, Melton LJ 3rd. Incidence of alopecia areata in Olmsted County, Minnesota, 1975 through 1989. Mayo Clin Proc. 1995;70:628-33.

2. Navarro R, Daudén E, Gallo E, Santiago Sánchez-Mateos D, García-Diez A. Alopecia areata during treatment of psoriasis with adalimumab and leflunomide: a case and review of the literature. Skin Pharmacol Physiol. 2012;25:107-10.

3. Garcia Bartels N, Lee HH, Worm M, Burmester GR, Sterry W, Blume-Peytavi U. Development of alopecia areata universalis in a patient receiving Adalimumab. Arch Dermatol. 2006;142:1654-5.

4. Posten W, Swan J. Recurrence of alopecia areata in a patient receiving etanercept injections. Arch Dermatol. 2005;141:759-60.

5. Tosti A, Pazzaglia M, Starace M, Bellavista S, Vincenzi C, Tonelli G. Alopeia Areata during treatment with biologic agents. Arch Dermatol. 2006;142:1653-4.

6. Zschoche C, Bidier M, Hadaschik E. Alopecia areata during treatment with adalimumabe: therapy with an alternative TNF-alpha inhibitor is possible. J Dtsch Dermatol Ges. 2013;11:450-3.

7. Gottenberg JE, Venancie PY, Mariette X. Alopecia areata in a patient with rheumatoid arthritis treated with leflunomide. J Rheumatol. 2002;29:1806-7.

8. Kubota T, Ishikura T, Jibiki I. Alopecia areata associated with haloperidol. Jpn J Psychiatry Neurol. 1994;48:579-81.

9. Chartaux E, Joly P. Long-term follow-up of the efficacy of methotrexate alone or in combination with low doses of oral corticosteroids in the treatment of alopecia areata totalis or universalis. Ann Dermatol Venereol. 2010;137:507-13.

\author{
MAILING ADDRESS: \\ Rosana Lazzarini \\ Rua Dr Cesario Mota Jr, 112 \\ Santa Cecilia \\ 01221-020 - São Paulo - SP \\ Brazil \\ E-mail: rosana.fototerapia@gmail.com
}

How to cite this article: Lazzarini R, Caparelli GC, Buense R, Lellis RF. Alopecia universalis during treatment with leflunomide and adalimumab - Case report. An Bras Dermatol. 2014;89(2):320-2. 